\title{
Modified Bat Algorithm for the Multi-Objective Flexible Job Shop Scheduling Problem
}

\author{
Haodong $\mathrm{Zhu}^{\mathrm{a}, \mathrm{b}}$, Baofeng $\mathrm{He}^{\mathrm{a}}$, Hongchan $\mathrm{Li}^{\mathrm{b}}{ }^{, *}$ \\ ${ }^{a}$ School of Electronic Information Engineering, Sias International University, Zhengzhou University, Xinzheng, Henan,451150, China \\ ${ }^{b}$ School of Computer and Communication Engineering, Zhengzhou University of Light Industry, Zhengzhou, Henan, 450002, China
}

\begin{abstract}
In this paper, a modified bat algorithm (MBA) is proposed for solving the multi-objective flexible job shop scheduling problem. Three different production performance indicators are considered which are the makespan, the total workload of machines and the critical machine workload. Firstly, to make the algorithm adaptive to the problem, the converting approaches are presented to implement the conversion between the continuous position vector and the discrete scheduling code. Secondly, an initialization scheme combining heuristics and random rule is introduced to ensure good quality and diversity of the initial population. Furthermore, five neighborhood structures are designed based on individual positions. Then, a local search algorithm is embedded into the BA to enhance the local searching ability. Finally, simulation results demonstrate the feasibility and effectiveness of our proposed algorithm.
\end{abstract}

Keywords: flexible job shop scheduling; multi-objective combinatorial optimization; bat algorithm; local search

(Submitted on July 21, 2017; Revised on September 8, 2017; Accepted on October 10, 2017)

(C) 2017 Totem Publisher, Inc. All rights reserved.

\section{Introduction}

Workshop scheduling considers a reasonable resource allocation to perform a collection of production tasks. It is viewed as an important decision-making process in most manufacturing industries. Job shop scheduling problem (JSSP) is one of the most difficult combinational optimization problems in the area of production scheduling that has been proved to be a NP-hard problem. For a classical JSSP, each job is processed with a fixed and known processing order through all machines to minimize a certain criterion. As a generalization version of the classic job shop scheduling problem (JSSP), the flexible job shop scheduling problem (FJSSP) provides a closer approximation to real-life production. In FJSSP, the restriction of machine availability is reduced; that is to say, each operation may be processed on more than one machine. Compared with the classic JSSP, the machine assignment to operations should be considered. Therefore, the complexity of the FJSSP is increased in a great extent, which has already been proved to be NP-hard.

In recent decades, the FJSSP has lots of researchers' attention. Due to its complexity, meta-heuristic algorithms have become a practical alternative of solving techniques. Saidi-Mehrabad and Fattahi [17] proposed a tabu search algorithm to solve the FJSSP with the objective of minimizing the makespan. Yazdani et al. [23] presented a parallel variable neighborhood search algorithm to deal with the FJSSP. Bagheri et al. [1] developed an artificial immune algorithm based on integrated approach. Wang et al. [20] proposed an artificial bee colony algorithm for solving the FJSSP. Rahmati and Zandieh [15] introduced a biogeography-based optimization algorithm for the FJSSP. Yuan et al. [26] designed a novel hybrid harmony search algorithm to solve the flexible job shop scheduling problem with the criterion of makespan minimization. As researchers probed more deeply and widely, they started to study the multi-objective FJSSP. Kaplanoğlu [8] presented an object-oriented (OO) approach for multi-objective FJSP along with simulated annealing optimization algorithm. Shivasankaran et al. [18] proposed a hybrid sorting immune simulated annealing technique (HSISAT) for solving 
the FJSSP. Xia et al. [22] developed a hybrid algorithm (PSO+SA) for the multi-objective FJSSP with the criterion to minimize the makespan, the total workload of machines and the workload of the critical machine. Wang et al [21] proposed a hybrid algorithm (ECO+TS) by combining the election campaign optimization and tabu search. Gao et al. [3] proposed a Pareto-based grouping discrete harmony search algorithm to deal with the flexible job shop scheduling problem considering two objectives: makespan and the mean of earliness and tardiness. Li et al. [10] developed a discrete artificial bee colony algorithm to solve the multi-objective FJSSP with maintenance activities.

In this paper, we proposed a modified bat algorithm (MBA) for solving the multi-objective flexible job shop scheduling problem. The basic bat algorithm is a new nature-inspired meta-heuristic algorithm, which is designed by Yang on the basis of the echolocation behavior of bats [24]. Since it has been proposed, bat algorithm has been used for solving various optimization problems $[4,16,25]$. However, in the manufacturing field, bat algorithm is seldom used for the production scheduling problem. Marichelvam and Prabaharam [12] proposed a bat algorithm for the hybrid flow shop scheduling problem. Marichelvam et al. [13] discussed the multistage hybrid flow shop scheduling problems using the bat algorithm. Luo et al. [11] developed a discrete bat algorithm to solve the permutation flow shop scheduling problem. Tosun and Marichelvam [19] integrated the local search into the bat algorithm to solve the permutation flow shop scheduling problems with the makespan criterion. In those studies, various scheduling problems in flow shops are considered. As far as we know, the bat algorithm is first introduced to deal with the multi-objective FJSSP in this paper.

The main contribution of this paper is to derive a modified bat algorithm (MBA) to solve the multi-objective flexible job shop scheduling problem. First, representation methods of scheduling solution and individual position vector are designed. Then, the conversion mechanism between the position vector and the scheduling scheme is presented to make the bat algorithm adaptive to discrete optimization algorithm. To improve the quality of the final solution, we propose a population initialization scheme and a variable neighborhood search algorithm. The computational results demonstrate that our proposed MBA is feasible and valid for the multi-objective FJSSP.

\section{Problem Formation}

In a FJSSP, $n$ jobs and $m$ machines are considered in the workshop. Jobs are independent with each other and have the same priorities. Each job consists of a certain sequence of operations whose processing times are determined by the machine assignment. The objective of the FJSSP aims to minimize three indicators, which are makespan ( $C_{\max }$ ), critical machine workload $\left(W_{\max }\right)$ and total workload of all machines $\left(W_{\mathrm{T}}\right)$. For such a system, some assumptions should be considered as follows:

(1) All machines and jobs are available at time 0.

(2) Each machine can perform only one operation simultaneously.

(3) The process of each operation can not be interrupted once it starts.

(4) Operations of each job must be processed after its predecessor is completed.

(5) Setup time of each machine is negligible.

To facilitate the establishment of the mathematical model, some symbols and variables are defined.

$n:$ the number of jobs;

$m:$ the number of machines;

$J_{i}:$ the number of operations of job $i$;

$O_{i j}:$ the $j$ th operation of job $i$;

$p_{i j k}$ : the processing time of $O_{i j}$ on machine $k$;

$S_{i j}:$ the start time of $O_{i j}$;

$C_{i j}:$ the complete time of $O_{i j}$;

$W_{k}$ : the workload on the machine $k$;

$\eta$ : a large positive number;

$x_{i j k}: 0-1$ variable, if $O_{i j}$ is processed on machine $k, x_{i j k}=1$; otherwise, $x_{i j k}=0$;

$y_{i j j^{\prime} j^{\prime} k}: 0-1$ variable, if $O_{i j}$ is processed on machine $k$ prior to $O_{i j^{\prime}}, y_{i j j^{\prime} j^{\prime} k}=1$; otherwise, $y_{i j j^{\prime} j^{\prime} k}=0$. 


$$
\begin{gathered}
\min C_{\max }=\min \left(\max \left(C_{i j}\right)\right) \\
\min W_{\mathrm{T}}=\min \left(\sum_{k=1}^{m} W_{k}\right) \\
\min W_{\max }=\min \left(\max \left(W_{k}\right)\right) \\
\text { s.t. } C_{i j}=S_{i j}+\sum_{k=1}^{m} x_{i j k} p_{i j k}, i=1,2, \cdots, n ; j=1,2, \cdots, J_{i} \\
S_{i(j+1)}-C_{i j} \geq 0, i=1,2, \cdots, n ; j=1,2, \cdots, J_{i}-1 \\
S_{i j^{\prime} j^{\prime}}+\eta\left(1-y_{i j i^{\prime} j^{\prime} k}\right) \geq C_{i j}, i, i^{\prime}=1,2, \cdots, n ; j, j^{\prime}=1,2, \cdots, J_{i} ; k=1,2, \cdots, m \\
S_{i j j}+\eta y_{i j i^{\prime} j^{\prime} k} \geq C_{i j^{\prime}}, i, i^{\prime}=1,2, \cdots, n ; j, j^{\prime}=1,2, \cdots, J_{i} ; k=1,2, \cdots, m \\
\sum_{k=1}^{m} x_{i j k}=1, i=1,2, \cdots, n ; j=1,2, \cdots, J_{i} \\
x_{i j k} \in\{0,1\}, i=1,2, \cdots, n ; j=1,2, \cdots, J_{i} ; k=1,2, \cdots, m \\
y_{i j i^{\prime} j^{\prime} k} \in\{0,1\}, i, i^{\prime}=1,2, \cdots, n ; j, j^{\prime}=1,2, \cdots, J_{i} ; k=1,2, \cdots, m
\end{gathered}
$$

Equations (1)-(3) refers to the optimization objective; constraint (4) guarantees that no preemption is allowed; constraint (5) ensures the precedence relationships between operations; constraint (6) and (7) show that every machine can only process one operation at a time; constraint (8) presents that each operation cannot be assigned to another machine once it starts; equations (9) and (10) gives $0-1$ variables.

For solving the multi-objective optimization problem, there exist many approaches, which can be classified into three types [9]: (1) the transformation approach, which transforms the multi-objective problem into a mono-objective one by introducing some weight coefficients; (2) the non-Pareto approach, which treats each objective in a separated way; (3) the Pareto approach, which is based on the Pareto optimization concept.

In this study, the objective function is determined by following the first approach, which is considered as the weighted sum of the three objectives, i.e., $\min f=w_{1} C_{\max }+w_{2} W_{\mathrm{T}}+w_{3} W_{\max }, w_{1}+w_{2}+w_{3}=1$.

\section{Basic Bat Algorithm}

The principle of the basic bat algorithm originated from the natural bat's echolocation behavior. In the algorithm, each individual is deemed as a micro-bat in a flock. On the premise of some approximate or idealized rules of the actual behavior of bats, the optimization objective is obtained by continuously updating the individual position and velocity vector based on the variation of pulse frequency, pulse emission rate and pulse loudness. The steps of the basic bat algorithm are listed below.

Step1. Initialize the population, set parameters and the terminate condition.

Step2. Evaluate each individual and find the best individual position vector $\boldsymbol{x}^{*}$.

Step3. Update the individual position and velocity vectors following Equations (11)-(13), where $f r_{\min }$ and $f r_{\max }$ are respectively the minimum and maximum values of the pulse frequency $f r_{i}, \boldsymbol{x}_{i}^{t}$ and $\boldsymbol{v}_{i}^{t}$ express the individual position and velocity vectors of bat $i$ in the $t$ th iteration, and $\beta \in[0,1]$.

$$
\begin{aligned}
& f r_{i}=f r_{\text {min }}+\left(f r_{\text {max }}-f r_{\text {min }}\right) \times \beta \\
& \boldsymbol{v}_{i}^{t}=\boldsymbol{v}_{i}^{t-1}+\left(\boldsymbol{x}_{i}^{t-1}-\boldsymbol{x}^{*}\right) f r_{i} \\
& \boldsymbol{x}_{i}^{t}=\boldsymbol{x}_{i}^{t-1}+\boldsymbol{v}_{i}^{t}
\end{aligned}
$$

Step 4. For each individual, a random number rand $\in[0,1]$ is generated. If $r a n d$ is greater than the pulse emission rate $r_{i}$, i.e., rand $>r_{i}$, a local search strategy is performed to the current best individual position vector to obtain a new position $\boldsymbol{x}_{i}^{\prime}$.

Step 5. Evaluate the new individual, and generate a random number rand $\in[0,1]$. If $r a n d$ is smaller than the loudness $A_{i}$, i.e., rand $<A_{i}$, and the objective value $f\left(\boldsymbol{x}_{i}^{\prime}\right)<f\left(\boldsymbol{x}^{*}\right)$, then $\boldsymbol{x}_{i}^{\prime}$ is accepted, the pulse emission rate $r_{i}$ and loudness $A_{i}$ are subsequently updated following Equations (14) and (15). $\lambda$ and $\gamma$ are constants which determine 
the variation of $r_{i}$ and $A_{i}$.

$$
\begin{aligned}
& A_{i}^{t+1}=\lambda A_{i}^{t} \\
& r_{i}^{t+1}=r_{i}^{0}(1-\exp (1-\gamma t))
\end{aligned}
$$

Step 6. Update the current best individual position vector $x^{*}$. If the terminate condition is met, go to Step 7, otherwise, go to Step3.

Step 7. Terminate the procedure.

\section{Modified Bat Algorithm}

\subsection{Scheduling Solution Representation}

In a FJSSP, machine assignment and operation permutation should be considered. The former aims to assign an appropriate machine to each operation, while the latter attempts to obtain a process sequence on each machine by maintaining the precedence constraints of operations. Therefore, in this study, a scheduling solution consists of two sections, whose length $q$ is equal to the total number of operations. The representation method is shown in Figure 1, where the first half section is the operation permutation, and the second half is the machine assignment. As seen from Figure 1, three jobs are involved, each of which contains two operations. In the first section, operations of the same job have the same element values, i.e., the second ' 2 ' means the 2 nd operation of Job 2. In the second section, each element value represents the code of the machine assigned

\begin{tabular}{|c|c|c|c|c|c|c|c|c|c|c|c|}
\hline$O_{11}$ & $O_{21}$ & $O_{31}$ & $O_{12}$ & $O_{22}$ & $O_{32}$ & $O_{11}$ & $O_{12}$ & $O_{21}$ & & $O_{31}$ & $O_{32}$ \\
\hline 1 & 2 & 3 & 1 & 2 & 3 & 2 & 1 & 1 & 2 & 3 & 3 \\
\hline
\end{tabular}
to the corresponding operation.

Figure 1. Scheduling solution representation

\subsection{Individual Position Vector}

\subsubsection{Position Vector Representation}

In the proposed MBA, each individual position vector is still a multi-dimensional real number vector, i.e., $\boldsymbol{x}=\{x(1), x(2), \cdots, x(l)\}$, where $x(j) \in[-\varepsilon, \varepsilon], j=1,2, \cdots, l$. According to the representation of the scheduling scheme, the position vector is also composed of two sections. The size of the vector equals the double of the total number of operations in the workshop, i.e., $l=2 q$. Figure 2 shows the representation of an individual position vector. The first half section describes the information of operation sequence, while the second half section shows the information of machine assignment. In the

\begin{tabular}{|c|c|c|c|c|c|c|c|c|c|c|c|}
\hline$O_{11}$ & $O_{12}$ & $O_{21}$ & $O_{22}$ & $O_{31}$ & $O_{32}$ & $O_{11}$ & $O_{12}$ & $O_{21}$ & $O_{22}$ & $O_{31}$ & $O_{32}$ \\
\hline-0.4 & 1.4 & -1.8 & 1.3 & 2.5 & -0.9 & 2.1 & 2.8 & -0.6 & 1.1 & -0.5 & 1.4 \\
\hline
\end{tabular}
two sections, element values are stored according to the given order, as shown in Figure 2.

Figure 2. Individual position vector

\subsubsection{Converting the Position Vector to the Scheduling Solution}

Due to the discrete characteristics of the FJSSP, the first issue in our algorithm is how to establish the mapping relationship between the individual position vector and the scheduling solution. The conversion mechanism is shown below.

(1) For the first half section, the real number position vector is supposed to be transformed into an operation permutation. First, the element values are rearranged in ascending order. According to the new order, the operation permutation can be obtained by reordering the operation codes according to the real numbers. Figure 3 shows an example of the conversion process. 


\begin{tabular}{|c|c|c|c|c|c|c|}
\hline Operation name & $O_{11}$ & $O_{12}$ & $O_{21}$ & $O_{22}$ & $O_{31}$ & $O_{32}$ \\
\hline \multirow[t]{2}{*}{ Individual position } & -0.4 & 1.4 & -1.8 & 1.3 & 2.5 & -0.9 \\
\hline & \multicolumn{6}{|c|}{5} \\
\hline Operation code & 1 & 1 & 2 & 2 & 3 & 3 \\
\hline \multirow[t]{2}{*}{ Individual position } & -0.4 & 1.4 & -1.8 & 1.3 & 2.5 & -0.9 \\
\hline & \multicolumn{6}{|c|}{$\sqrt{3}$} \\
\hline $\begin{array}{c}\text { Corresponding } \\
\text { operation }\end{array}$ & 2 & 3 & 1 & 2 & 1 & 3 \\
\hline \multirow[t]{2}{*}{ Ascending order } & -1.8 & -0.9 & -0.4 & 1.3 & 1.4 & 2.5 \\
\hline & \multicolumn{6}{|c|}{ 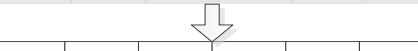 } \\
\hline Operation permutation & 2 & 3 & 1 & 2 & 1 & 3 \\
\hline
\end{tabular}

Figure 3. The conversion from an individual position vector to an operation permutation

(2) For the second half section, the real number vector will be transformed into a machine assignment. The conversion formula is based on the approach proposed by Yuan et al. [6].

$$
u(h)=\operatorname{round}\left(\frac{\varepsilon+x(h+q)}{2 \varepsilon}(z(h)-1)+1\right), 1 \leq h \leq q
$$

In Equation (16), $z(h)$ denotes the size of the alternative machine set of the operation corresponding to the $h+q$ th element in the individual position vector. $u(h) \in[1, z(h)]$ is the index of the selected machine in the alternative machine set. round () is the function that rounds the number to the nearest integer.

\subsection{Initial Scheduling Solution}

The population of initial scheduling solutions is a crucial factor which affects the convergence speed and the solution quality in a great extent. According to the two sub-problems in FJSSP, initial scheduling solutions are generated by considering for machine assignment part and operation sequencing part.

\subsubsection{Machine Assignment Initialization}

The machine assignment component considers how to assign an appropriate machine to each operation. By considering both the problem features and the objectives, global selection (GS), local selection (LS) and random selection (RS) are adopted which are proposed by Zhang et al. [27]. The GS could better explore the search space by acquiring various initial assignments in different runs. The LS could find the machine with the shortest processing time in alternative machine set of each job. To ensure the diversity of initial population, the RS is used to generate initial assignments. In this paper, $60 \%$ of initial solutions are generated by GS, $20 \%$ by LS, and $20 \%$ by RS.

\subsubsection{Operation Sequencing Initialization}

The operation sequencing component considers how to sequence the operations assigned to each machine and determine the start/completion time of each operation. Five dispatching rules are used as follows:

Shortest Processing Time (SPT): The operation with the minimal processing time has the highest priority to be processed. Longest Processing Time (LPT): The operation with the maximal processing time has the highest priority to be processed. Most Work Remaining (MWR): The job with the maximal total processing time has the highest priority to be processed. Most Operation Remaining (MOR): The job with the most remaining operations has the highest priority to be processed. Random Rule (RR): The operation sequence is obtained by a random permutation of operations of all jobs.

\subsubsection{Population Initialization}

As mentioned above, the scheduling solution consists of two parts: machine assignment and operation sequencing. To initial the population of the algorithm, the machine assignment components are first generated by using the methods in Section 4.3.1. For each machine assignment, a predefined number of operation permutations are randomly generated according to the above 
five rules. Then, the best combination of the machine assignment and its candidate operation permutation is set to be an initial scheduling solution and added to the initial population. The iteration is repeated until all the initial scheduling solutions have been generated.

\subsubsection{Converting the Scheduling Solution to the Position Vector}

By considering the searching mechanism of the bat algorithm, the generated initial scheduling schemes should be converted to continuous position vectors. This conversion also consists of two sections in Section 4.2.

(1) For the first part related to operation sequence, the conversion process is illustrated in Figure 4 . Firstly, $0.5 \mathrm{l}$ real numbers are randomly generated in $[-\varepsilon, \varepsilon]$. Then these numbers are rearranged in an ascending order and corresponded to operations in the initial permutation. The individual position vector is determined by reordering the real numbers according to the operation codes.

\begin{tabular}{|c|c|c|c|c|c|c|}
\hline \multirow[t]{2}{*}{ Random number } & 1.4 & -0.5 & -2.8 & 1.3 & 2.5 & 1.7 \\
\hline & \multicolumn{6}{|c|}{ 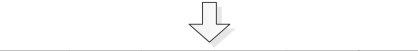 } \\
\hline Ascending order & -2.8 & -0.5 & 1.3 & 1.4 & 1.7 & 2.5 \\
\hline \multirow[t]{2}{*}{ Initial permutation } & 2 & 3 & 1 & 2 & 1 & 3 \\
\hline & \multicolumn{6}{|c|}{3} \\
\hline Operation code & 1 & 1 & 2 & 2 & 3 & 3 \\
\hline Individual position & 1.3 & 1.7 & -2.8 & 1.4 & -0.5 & 2.5 \\
\hline
\end{tabular}

Figure 4. The conversion from an initial scheduling solution to an individual position vector

(2) For the second part, the conversion formula (17) proposed in [6] is used, which is in fact an inverse version of Equation (16). But when $z(h)=1, x(h)$ should be random selected in $[-\varepsilon, \varepsilon]$.

$$
x(h+q)= \begin{cases}\frac{2 \varepsilon}{z(h)-1}(u(h)-1)-\varepsilon, & z(h) \neq 1 \\ x(h+q) \in[-\varepsilon, \varepsilon], & z(h)=1\end{cases}
$$

\subsection{Local Search}

To further improve the local search capacity, a local search (LS) algorithm is performed on the current best individual of MBA during the searching process. In the LS, five neighborhood structures are systematically changed to make the algorithm escape from the local optima.

\subsubsection{Neighborhood Structure}

The neighborhood structures are designed for the searching process as below.

(1) Operation permutation neighborhood

Neighborhood $N_{1}$ : Randomly select two elements $e_{1}$ and $e_{2}$ corresponding to different jobs in the position vector, and then swap $e_{1}$ with $e_{2}$.

Neighborhood $N_{2}$ : Randomly select two elements $e_{1}$ and $e_{2}$ corresponding to different jobs in the position vector, and then insert $e_{2}$ before $e_{1}$.

Neighborhood $N_{3}$ : Randomly select two elements $e_{1}$ and $e_{2}$ corresponding to different jobs in the position vector, and then inverse the items between $e_{1}$ and $e_{2}$.

(2) Machine assignment neighborhood

Neighborhood $N_{4}$ : Randomly select an element in the second section of the candidate position vector, which corresponds to an operation with more than one alternative machine. A machine is selected from alternative machines to replace the current one. A new real number will be regenerated by Equation (7) to replace the current value of the selected element. 
Neighborhood $N_{5}$ : Randomly select an element in the second section of the candidate position vector, which corresponds to an operation with more than one alternative machine. The current machine for the selected operation will be replaced by the one with the shortest processing time among alternative machines. A new real number will be regenerated by Equation (7) to replace the current value of the selected element.

\subsubsection{Step of the $L S$}

The local search is constructed by randomly selecting one approach from the three operation permutation neighborhoods and one method from the machine assignment neighborhoods. The procedure starts from a given solution and stops when the maximum iteration is met. The procedure of the local search is given as below.

Step 1. Initialization. Acquire the initial position vector $\boldsymbol{x}$, set the terminate condition $\rho_{\max }$ and $\rho \leftarrow 0$.

Step 2. while $\rho<\rho_{\max }$

$x^{\prime}:=$ Randomly perform an operation permutation neighborhood and a machine assignment neighborhood to $x$

if $f\left(\boldsymbol{x}^{\prime}\right)<f(\boldsymbol{x})$ then $\boldsymbol{x}:=\boldsymbol{x}^{\prime}$

end if

$\rho \leftarrow \rho+1$

end while

Step 3. Obtain the local optima $\boldsymbol{x}$.

\subsection{Updating Method of Pulse Emission Rate and Loudness}

The initial pulse emission rate $r_{i}$ is set to be a positive and small value. With the iteration process conducting, $r_{i}$ will be increased to 1. In this paper, the updating method of $r_{i}$ follows Equation (18) with a curve similar with the one of Sigmoid function [19].

$$
r_{i}(t)=\left(1+\exp \left(-\frac{10}{t_{\max }} \times\left(t-\frac{t_{\max }}{2}\right)+r_{i}^{0}\right)\right)^{-1}
$$

By this formula, the algorithm may exploit near the current best individual position with a large probability to speed up the convergence process in the early iteration. In the later searching stage, the diversity of the algorithm can be guaranteed to avoid the premature [19]. For the loudness $A_{i}$, the updating method is expressed by Equation (19), where $f_{i}$ denotes the objective value of individual $i, f_{\max }$ and $f_{\min }$ are the maximum and the minimum objective values in the current population.

\subsection{Steps of $M B A$}

$$
A_{i}=\frac{f_{i}-f_{\min }}{f_{\max }-f_{\min }}
$$

The detailed steps of MBA are listed below.

Step 1. Initialization. Set related parameters of the algorithm.

Step 2. Generate the initial population, evaluate each individual, and find the current best individual position vector $\boldsymbol{x}^{*}$.

Step 3. Update the individual position and velocity according to Equations (11)-(13).

Step 4. For each individual, if rand $>r_{i}$, the local search is performed to the current best individual to generate a new position $\boldsymbol{x}_{i}^{\prime}$.

Step 5. Evaluate the new individual, if rand $<A_{i}$ and $f\left(\boldsymbol{x}_{i}^{\prime}\right)<f\left(\boldsymbol{x}^{*}\right)$, then $\boldsymbol{x}_{i}^{\prime}$ is accepted, the pulse emission rate $r_{i}$ and loudness $A_{i}$ are subsequently updated following Equations (18) and (19).

Step 6. Update the current best individual position vector, and perform the local search to it to acquire a new one $\boldsymbol{x}^{*}$.

Step 7. Check the terminate condition $t_{\max }$. If it is met, go to Step 8, otherwise, go to Step 3.

Step 8. End the procedure. 


\section{Experiment Validations}

\subsection{Parameter Settings}

The benchmark instances taken from Kacem [6,7] and Brandimarte [2] are used to evaluate the performance of our algorithm. Parameters are set by comparison of computational results under different combinations, which are shown as follows: the population size is 100; the terminate conditions $t_{\max }=200$ and $\rho_{\max }=10$. For the objective considered in this paper, the three weights reflect the importance of each objective, which are determined based on the judgments of users. All computational results in this paper are obtained based on six different combinations of weights which are summarized in Table 1. For each weight set, 10 trials were conducted to obtain the experimental results.

Table 1. Different Weight Sets for the Objectives

\begin{tabular}{|c|c|c|c|}
\hline Weight set & $w_{1}$ & $w_{2}$ & $w_{3}$ \\
\hline 1 & 0.2 & 0.3 & 0.5 \\
\hline 2 & 0.3 & 0.5 & 0.2 \\
\hline 3 & 0.3 & 0.2 & 0.5 \\
\hline 4 & 0.4 & 0.4 & 0.2 \\
\hline 5 & 0.5 & 0.3 & 0.2 \\
\hline 6 & 0.7 & 0.1 & 0.2 \\
\hline
\end{tabular}

\subsection{Results Comparisons}

To verify the effectiveness, the proposed MBA is compared with other algorithms in existing literatures, such as OO [8], HSISAT [18], PSO+SA [22], ECO+TS [21], FL+EA [7], MOPSO+LS [6], TS [2] and MATLSO [5].

\subsubsection{The Five Kacem Instances}

\subsubsection{Problem $4 \times 5$}

Table 2. The Results of Kacem $1(4 \times 5)$

\begin{tabular}{|c|c|c|c|c|}
\hline Weight set & $f$ & $C_{\max }$ & $W_{\mathrm{T}}$ & $W_{\max }$ \\
\hline 1 & 16.0 & 12 & 32 & 8 \\
\hline \multirow{2}{*}{2} & 21.2 & 12 & 32 & 8 \\
\hline & 21.3 & 11 & 32 & 10 \\
\hline \multirow{2}{*}{3} & 14.0 & 12 & 32 & 8 \\
\hline & 14.7 & 11 & 32 & 10 \\
\hline \multirow{2}{*}{4} & 19.2 & 12 & 32 & 8 \\
\hline & 19.2 & 11 & 32 & 10 \\
\hline 5 & 17.1 & 11 & 32 & 10 \\
\hline \multirow{2}{*}{6} & 12.9 & 11 & 32 & 10 \\
\hline & 12.9 & 11 & 34 & 9 \\
\hline \multirow{3}{*}{$\begin{array}{l}\text { Optimal } \\
\text { solution }\end{array}$} & Solution 1 & 12 & 32 & 8 \\
\hline & Solution 2 & 11 & 32 & 10 \\
\hline & Solution 3 & 11 & 34 & 9 \\
\hline
\end{tabular}

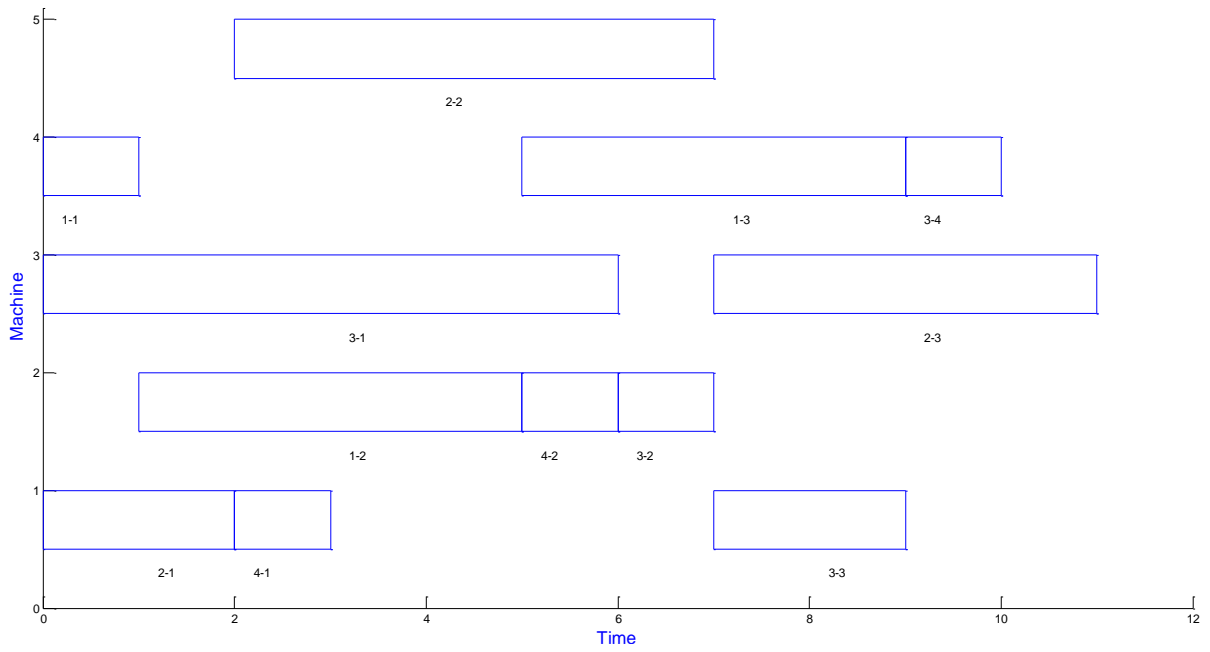

Figure 5. The Gantt chart of Solution 2 for Kacem1 (Problem $4 \times 5$ ) 
We first use a small-size instance to test the effectiveness of our algorithm in which 4 jobs with 12 operations are to be performed on 5 machines. The computational results obtained by our MBA are shown in Table 2. Figure 5 shows the result of Solution 2 in the form of a Gantt chart. Each rectangle represents an operation, under which the character denotes the name of the operation, i.e., 2-2 refers to the second operation of Job 2.

\subsubsection{Problem $8 \times 8$}

This is a middle-size instance in which 8 jobs with 27 operations are to be processed on 8 machines. The computational results obtained by our proposed algorithm are characterized by the following values in Table 3. Figure 6 shows the result of Solution 1 in the form of a Gantt chart.

Table 3. The Results of Kacem2 $(8 \times 8)$

\begin{tabular}{|c|c|c|c|c|}
\hline Weight set & $f$ & $C_{\max }$ & $W_{\mathrm{T}}$ & $W_{\max }$ \\
\hline \multirow{2}{*}{1} & 31.5 & 15 & 75 & 12 \\
\hline & 31.6 & 16 & 73 & 13 \\
\hline \multirow{2}{*}{2} & 43.9 & 16 & 73 & 13 \\
\hline & 44.4 & 15 & 75 & 12 \\
\hline \multirow{2}{*}{3} & 25.5 & 15 & 75 & 12 \\
\hline & 25.9 & 16 & 73 & 13 \\
\hline \multirow{2}{*}{4} & 38.2 & 16 & 73 & 13 \\
\hline & 38.4 & 15 & 75 & 12 \\
\hline \multirow{3}{*}{5} & 32.5 & 16 & 73 & 13 \\
\hline & 32.4 & 15 & 75 & 12 \\
\hline & 32.5 & 14 & 77 & 12 \\
\hline \multirow{3}{*}{6} & 21.1 & 16 & 73 & 13 \\
\hline & 20.4 & 15 & 75 & 12 \\
\hline & 19.9 & 14 & 77 & 12 \\
\hline \multirow{3}{*}{$\begin{array}{l}\text { Optimal } \\
\text { solution }\end{array}$} & Solution 1 & 16 & 73 & 13 \\
\hline & Solution 2 & 15 & 75 & 12 \\
\hline & Solution 3 & 14 & 77 & 12 \\
\hline
\end{tabular}

\subsubsection{Problem $10 \times 7$}

This is a middle-size instance in which 10 jobs with 29 operations are to be processed on 7 machines. The computational results obtained by our proposed algorithm are characterised by the following values in Table 4 . Figure 7 shows the result of Solution 2 in the form of a Gantt chart.

Table 4. The Results of Kacem3 $(10 \times 7)$

\begin{tabular}{|c|c|c|c|c|}
\hline $\begin{array}{c}\text { Weight } \\
\text { set }\end{array}$ & $f$ & $C_{\max }$ & $W_{\mathrm{T}}$ & $W_{\max }$ \\
\hline \multirow{3}{*}{1} & 26.4 & 12 & 60 & 12 \\
\cline { 2 - 5 } & 26.0 & 11 & 61 & 11 \\
\hline \multirow{3}{*}{2} & 36.0 & 12 & 60 & 12 \\
\hline \multirow{3}{*}{3} & 36.0 & 11 & 61 & 11 \\
\cline { 2 - 5 } & 21.0 & 11 & 61 & 11 \\
\cline { 2 - 5 } & 21.6 & 12 & 60 & 12 \\
\hline \multirow{3}{*}{5} & 20.7 & 11 & 62 & 10 \\
\cline { 2 - 5 } & 31.2 & 12 & 60 & 11 \\
\hline \multirow{2}{*}{6} & 31.0 & 11 & 61 & 12 \\
\cline { 2 - 5 } & 26.4 & 12 & 60 & 12 \\
\hline \multirow{2}{*}{$\begin{array}{c}\text { Optimal } \\
\text { solution }\end{array}$} & 16.8 & 11 & 61 & 11 \\
\cline { 2 - 5 } & Solution 1 & 12 & 60 & 11 \\
\cline { 2 - 5 } & Solution 2 & 11 & 61 & 12 \\
\hline
\end{tabular}




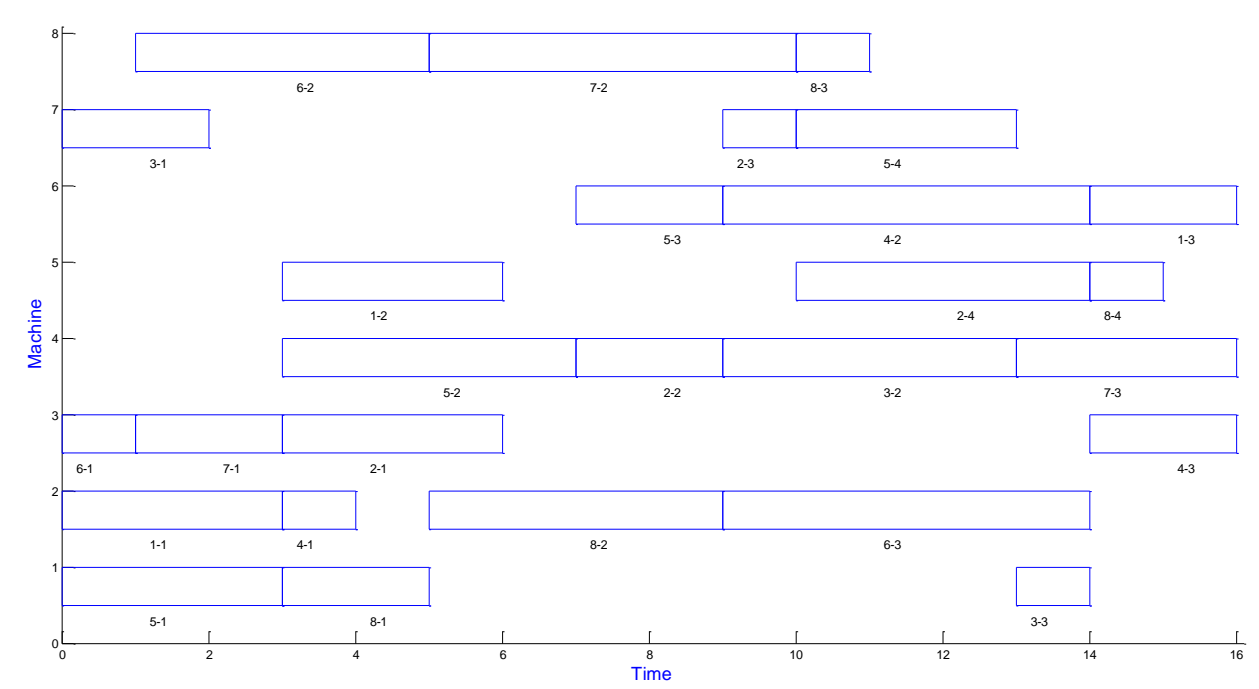

Figure 6. The Gantt chart of Solution 1 for Kacem2 (Problem $8 \times 8$ ).

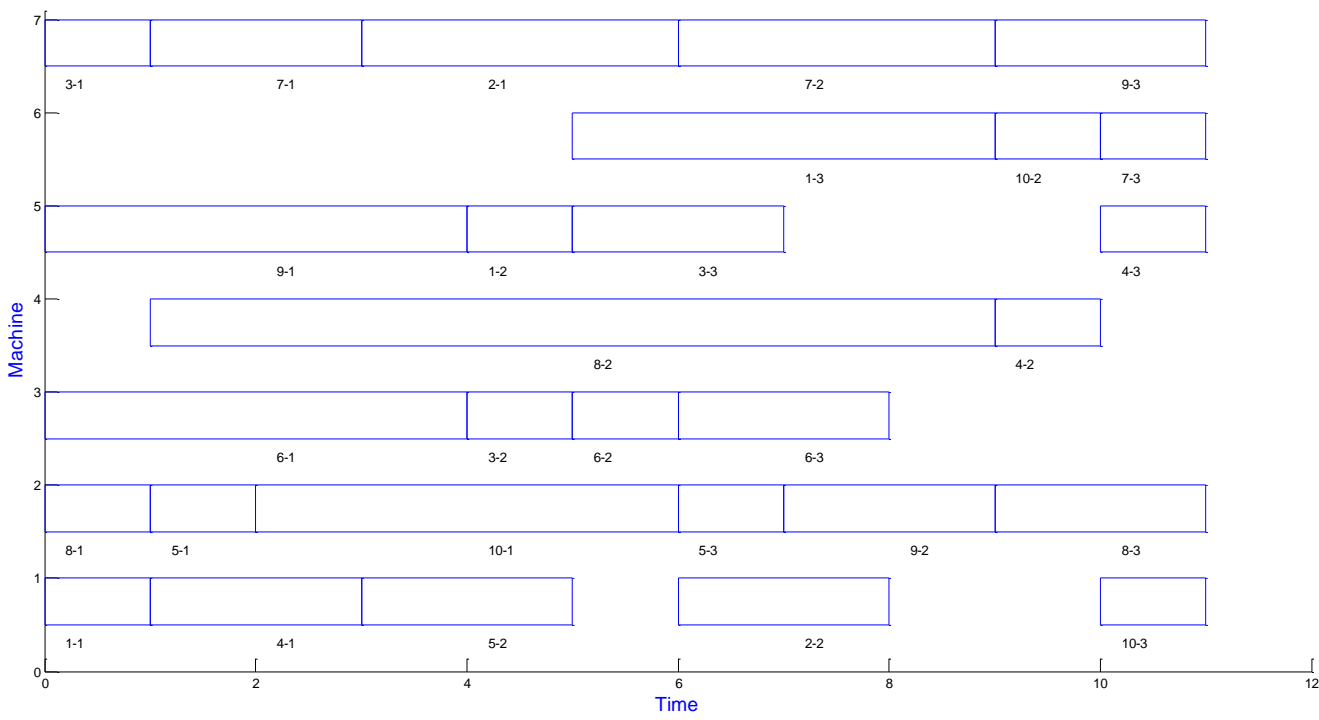

Figure 7. The Gantt chart of Solution 2 for Kacem 3 (Problem $10 \times 7$ ).

\subsubsection{Problem $10 \times 10$}

This is a middle-size instance in which 10 jobs with 30 operations are to be processed on 10 machines. The computational results obtained by our proposed algorithm are characterized by the following values in Table 5. Figure 8 shows the result of Solution 3 in the form of a Gantt chart.

Table 5. The Results of Kacem4 $(10 \times 10)$

\begin{tabular}{|c|c|c|c|c|}
\hline $\begin{array}{c}\text { Weight } \\
\text { set }\end{array}$ & $f$ & $C_{\max }$ & $W_{\mathrm{T}}$ & $W_{\max }$ \\
\hline \multirow{4}{*}{1} & 17.0 & 8 & 43 & 5 \\
\cline { 2 - 5 } & 17.2 & 8 & 42 & 6 \\
\cline { 2 - 5 } & 17.1 & 7 & 44 & 5 \\
\hline \multirow{3}{*}{2} & 17.4 & 8 & 41 & 7 \\
\hline \multirow{3}{*}{3} & 24.3 & 8 & 41 & 7 \\
\cline { 2 - 5 } & 24.6 & 8 & 42 & 6 \\
\cline { 2 - 5 } & 13.3 & 8 & 42 & 5 \\
\hline \multirow{3}{*}{4} & 13.5 & 7 & 42 & 6 \\
\hline \multirow{3}{*}{5} & 13.4 & 7 & 44 & 5 \\
\cline { 2 - 5 } & 21.0 & 8 & 41 & 7 \\
\cline { 2 - 5 } & 21.2 & 8 & 42 & 6 \\
\hline
\end{tabular}




\begin{tabular}{|c|c|c|c|c|}
\hline & 17.5 & 7 & 42 & 7 \\
\hline \multirow{4}{*}{6} & 10.9 & 8 & 43 & 5 \\
\cline { 2 - 5 } & 10.3 & 7 & 42 & 6 \\
\cline { 2 - 5 } & 10.3 & 7 & 44 & 5 \\
\cline { 2 - 5 } & 11.1 & 8 & 41 & 7 \\
\hline \multirow{3}{*}{$\begin{array}{c}\text { Optimal } \\
\text { solution }\end{array}$} & Solution 1 & 8 & 42 & 5 \\
\cline { 2 - 5 } & Solution 2 & 7 & 42 & 6 \\
\cline { 2 - 5 } & Solution 3 & 7 & 44 & 5 \\
\cline { 2 - 5 } & Solution 4 & 8 & 41 & 7 \\
\hline
\end{tabular}

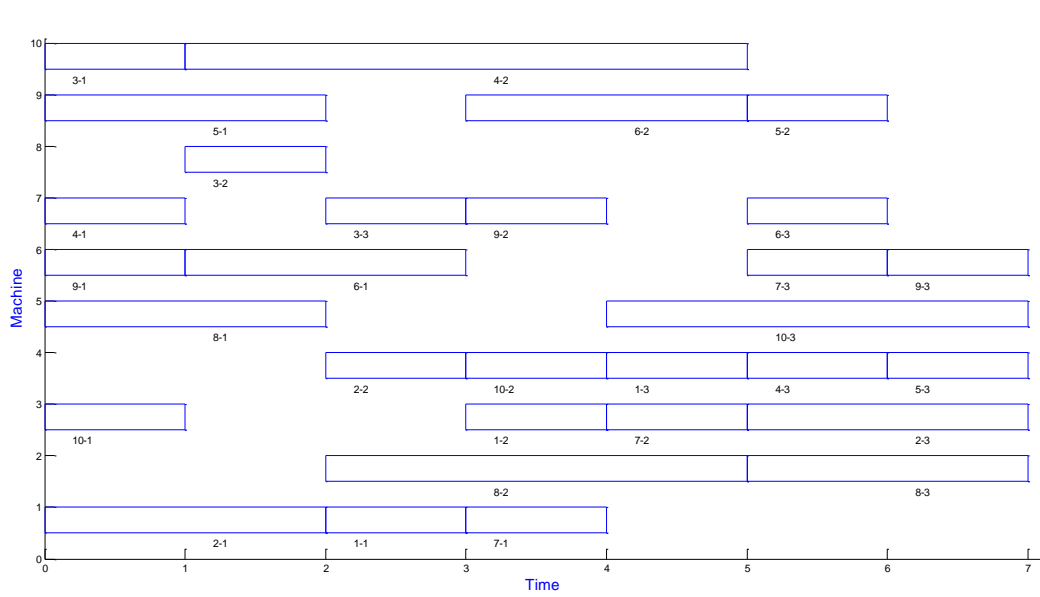

Figure 8. The Gantt chart of Solution 3 for Kacem4 (Problem $10 \times 10$ ).

\subsubsection{Problem $15 \times 10$}

This is a large-size instance in which 15 jobs with 56 operations are to be processed on 10 machines. The computational results obtained by our proposed algorithm are characterised by the following values in Table 6 . Figure 9 shows the result of Solution 1 in the form of a Gantt chart.

Table 7 shows the comparison results of the five Kacem instances, where '-' means that the corresponding result is not reported in the related literature, and the boldface denotes the best solutions obtained by algorithms. It can be seen from Table 7 that MBA is comparable to other algorithms for solving the Kacem instances. The comparison data point out that the proposed MBA can obtain all the non dominated solutions in Kacem instances 1, 3 and 4. For the Kacem 2, MBA can obtain richer optimal solutions than OO [7], HSISAT [8], PSO+SA [9], and ECO+TS [10]. For the Kacem5 instance, approximated non-dominated solutions were acquired by MBA. Table 7 shows that the proposed MBA algorithm performed at the same level or better with respect to three objective functions for the first four instances, when compared to the results obtained from the other methods.

Table 6. The Results of Kacem5 $(15 \times 10)$

\begin{tabular}{|c|c|c|c|c|}
\hline Weight set & $f$ & $C_{\max }$ & $W_{\mathrm{T}}$ & $W_{\max }$ \\
\hline \multirow{3}{*}{1} & 37.2 & 13 & 97 & 11 \\
\cline { 2 - 5 } & 35.9 & 14 & 92 & 11 \\
\hline \multirow{3}{*}{3} & 52.0 & 13 & 91 & 13 \\
\cline { 2 - 5 } & 52.6 & 14 & 92 & 12 \\
\cline { 2 - 5 } & 28.0 & 13 & 93 & 11 \\
\hline \multirow{3}{*}{4} & 28.9 & 15 & 92 & 12 \\
\cline { 2 - 5 } & 44.6 & 14 & 91 & 13 \\
\cline { 2 - 5 } & 45.2 & 15 & 92 & 12 \\
\cline { 2 - 5 } & 44.8 & 13 & 93 & 12 \\
\hline \multirow{3}{*}{5} & 45.0 & 14 & 93 & 11 \\
\cline { 2 - 5 } & 36.3 & 13 & 92 & 11 \\
\cline { 2 - 5 } & 20.9 & 13 & 94 & 11 \\
\hline \multirow{3}{*}{$\begin{array}{c}\text { Optimal } \\
\text { solution }\end{array}$} & 21.1 & 13 & 98 & 13 \\
\cline { 2 - 5 } & 21.6 & 14 & 96 & 92 \\
\hline
\end{tabular}




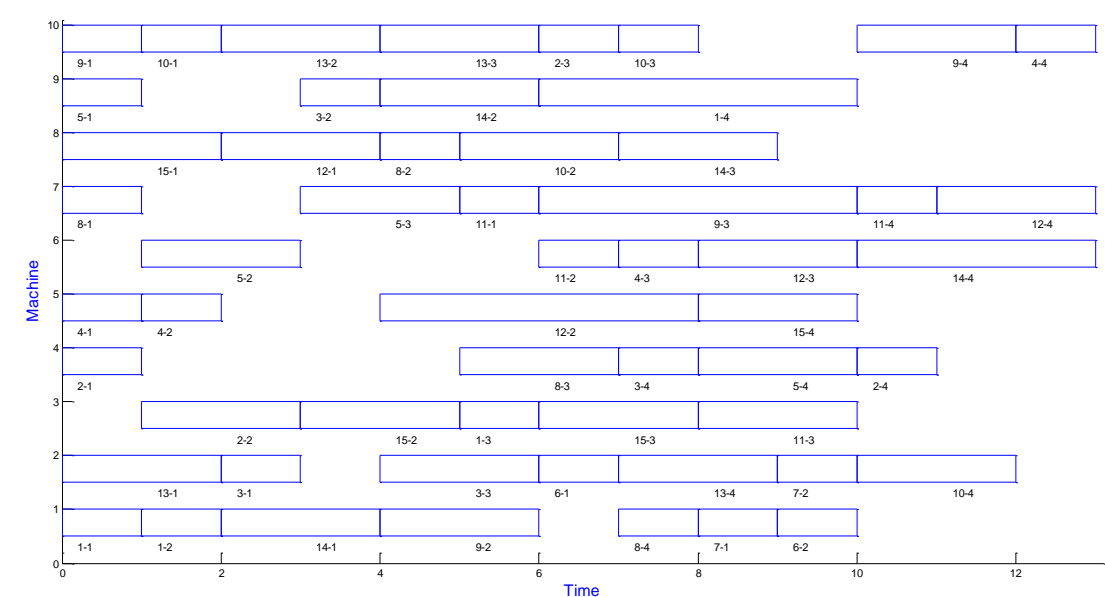

Figure 9. The Gantt chart of Solution 1 for Kacem 5 (Problem $15 \times 10$ ).

Table 7. The Comparison Results of Different Algorithms

\begin{tabular}{|c|c|c|c|c|c|c|c|c|c|c|c|c|c|c|c|c|c|c|}
\hline Instance & $n \times m$ & Objective & \multicolumn{3}{|c|}{$\mathrm{OO}$} & \multirow{2}{*}{$\begin{array}{c}\text { HSISAT } \\
\mathbf{1 2}\end{array}$} & \multicolumn{2}{|c|}{$\begin{array}{c}\mathrm{PSO}+\mathrm{S} \\
\mathrm{A}\end{array}$} & \multicolumn{2}{|c|}{$\begin{array}{c}\mathrm{ECO}+\mathrm{T} \\
\mathrm{S}\end{array}$} & \multicolumn{4}{|c|}{ MOGA } & \multicolumn{4}{|c|}{ MBA } \\
\hline \multirow{3}{*}{ Kacem1 } & \multirow{3}{*}{$4 \times 5$} & $C_{\text {max }}$ & 11 & - & - & & - & - & 11 & 11 & - & - & - & - & 11 & 12 & 11 & - \\
\hline & & $W_{\mathrm{T}}$ & 32 & - & - & 32 & - & - & 34 & 32 & - & - & - & - & 32 & 32 & 34 & - \\
\hline & & $W_{\max }$ & 10 & - & - & 8 & - & - & 9 & 10 & - & - & - & - & 10 & 8 & 9 & - \\
\hline \multirow{3}{*}{ Kacem2 } & \multirow{3}{*}{$8 \times 8$} & $C_{\max }$ & 16 & 15 & - & 16 & 15 & 16 & 14 & 15 & 15 & 15 & 16 & - & 16 & 15 & 14 & - \\
\hline & & $W_{\mathrm{T}}$ & 73 & 75 & - & 73 & 75 & 73 & 77 & 75 & 81 & 75 & 73 & - & 73 & 75 & 77 & - \\
\hline & & $W_{\max }$ & 13 & 12 & - & 13 & 12 & 13 & 12 & 12 & 11 & 12 & 13 & - & 13 & 12 & 12 & - \\
\hline \multirow{3}{*}{ Kacem3 } & \multirow{3}{*}{$10 \times 7$} & $C_{\max }$ & - & - & - & 16 & - & - & - & - & - & - & - & - & 12 & 11 & 11 & - \\
\hline & & $W_{\mathrm{T}}$ & - & - & - & 60 & - & - & - & - & - & - & - & - & 60 & 61 & 62 & - \\
\hline & & $W_{\max }$ & - & - & - & 12 & - & - & - & - & - & - & - & - & 12 & 11 & 10 & - \\
\hline \multirow{3}{*}{ Kacem4 } & \multirow{3}{*}{$10 \times 10$} & $C_{\max }$ & 8 & 8 & 7 & 8 & 7 & - & 7 & 8 & 7 & 8 & 8 & 7 & 8 & 7 & 8 & 7 \\
\hline & & $W_{\mathrm{T}}$ & 41 & 42 & 43 & 41 & 44 & - & 43 & 42 & 45 & 42 & 41 & 42 & 42 & 42 & 41 & 44 \\
\hline & & $W_{\text {max }}$ & 7 & 5 & 7 & 7 & 6 & - & 6 & 5 & 5 & 5 & 7 & 6 & 5 & 6 & 7 & 5 \\
\hline \multirow{3}{*}{ Kacem5 } & \multirow{3}{*}{$15 \times 10$} & $C_{\max }$ & 13 & 14 & - & 13 & 12 & - & - & - & 11 & 12 & 11 & - & 13 & 13 & - & - \\
\hline & & $W_{\mathrm{T}}$ & 91 & 91 & - & 91 & 91 & - & - & - & 91 & 95 & 98 & - & 92 & 91 & - & - \\
\hline & & $W_{\max }$ & 13 & 12 & - & 12 & 11 & - & - & - & 11 & 10 & 10 & - & 11 & 13 & - & - \\
\hline
\end{tabular}

\subsubsection{The Three Kacem Instances with Release Dates}

The second test compares the performances of FL+EA [7], MOPSO+LS [14] and our MBA on three instances with release dates for jobs. These instances were presented by Kacem et al. [7]. Table 2 indicates the comparison of the solutions acquired by three approaches, where '-' means that the corresponding result is not reported in the related literature.

Table 8. The Comparison Results of Instances with Release Dates

\begin{tabular}{|c|c|c|c|c|c|c|c|c|c|c|c|c|c|}
\hline Instance & $n \times m$ & Objective & \multicolumn{5}{|c|}{ FL+EA } & \multicolumn{3}{|c|}{ MOPSO+LS } & \multicolumn{3}{|c|}{ MBA } \\
\hline \multirow{3}{*}{ Kacem1 } & \multirow{3}{*}{$4 \times 5$} & $C_{\max }$ & 18 & 18 & 16 & 16 & - & 16 & 16 & - & 16 & 16 & - \\
\hline & & $W_{\mathrm{T}}$ & 32 & 33 & 35 & 34 & - & 32 & 33 & - & 32 & 33 & - \\
\hline & & $W_{\max }$ & 8 & 7 & 9 & 10 & - & 8 & 7 & - & 8 & 7 & - \\
\hline \multirow{3}{*}{ Kacem3 } & \multirow{3}{*}{$10 \times 7$} & $C_{\max }$ & 16 & 15 & 18 & 17 & 16 & 16 & 15 & 15 & 16 & 15 & 15 \\
\hline & & $W_{\mathrm{T}}$ & 60 & 61 & 63 & 64 & 66 & 60 & 61 & 62 & 60 & 61 & 62 \\
\hline & & $W_{\max }$ & 12 & 11 & 10 & 10 & 10 & 12 & 11 & 10 & 12 & 11 & 10 \\
\hline \multirow{3}{*}{ Kacem5 } & \multirow{3}{*}{$15 \times 10$} & $C_{\max }$ & 24 & 23 & - & - & - & 23 & - & - & 23 & 23 & 25 \\
\hline & & $W_{\mathrm{T}}$ & 91 & 95 & - & - & - & 91 & - & - & 92 & 93 & 91 \\
\hline & & $W_{\max }$ & 11 & 11 & - & - & - & 11 & - & - & 12 & 11 & 11 \\
\hline
\end{tabular}


It can be observed from Table 8 that the computational results of the proposed algorithm dominate the results of the FL+ EA for solving the Kacem 1 and 3 instances. In addition, three approximated solutions were obtained in Kacem5. The release dates of jobs are listed as follows:

(1) Kacem 1: release dates: $r_{1}=3, r_{2}=5, r_{3}=1, r_{4}=6$.

(2) Kacem 3: release dates: $r_{1}=2, r_{2}=4, r_{3}=9, r_{4}=6, r_{5}=7, r_{6}=5, r_{7}=7, r_{8}=4, r_{9}=1, r_{10}=0$.

(3) Kacem 5: release dates: $r_{1}=5, r_{2}=3, r_{3}=6, r_{4}=4, r_{5}=9, r_{6}=7, r_{7}=1, r_{8}=2, r_{9}=9, r_{10}=0, r_{11}=14, r_{12}=13$,

$$
r_{13}=11, r_{14}=12, r_{15}=5 \text {. }
$$

\subsubsection{The BRdata Instances}

For further comparison, ten BRdata instances MK1 MK10 taken from Brandimarte [2] are used to compare the makespan obtained by algorithms. Two published algorithms are compared with our algorithm, i.e., TS [2] and MATLSO [5]. Table 9 suggests that the best makespan of our approach is superior to the TS algorithm for 9 ount of 10 cases. Regarding the approach MATSLO and MBA, the optimal makespan of MBA is superior to that of MATSLO for 7 out of 10 cases. To summarize, the comparison results show that our proposed algorithm outperforms most of these published algorithms, and is competent for the flexible job shop scheduling problems.

\begin{tabular}{|c|c|c|c|c|}
\multicolumn{6}{c}{ Table 9. The Comparison Results of two Algorithms } \\
\cline { 2 - 5 } & \multirow{2}{*}{ Instance } & TS & MATSLO & MBA \\
\cline { 3 - 5 } & & Best & Best & Best \\
\hline MK1 & $10 \times 6$ & 42 & $\mathbf{4 0}$ & 42 \\
\hline MK2 & $10 \times 6$ & 32 & 32 & $\mathbf{3 0}$ \\
\hline MK3 & $15 \times 8$ & 211 & 207 & $\mathbf{2 0 4}$ \\
\hline MK4 & $15 \times 8$ & 81 & $\mathbf{6 7}$ & 71 \\
\hline MK5 & $15 \times 4$ & 186 & 188 & $\mathbf{1 8 1}$ \\
\hline MK6 & $10 \times 15$ & 86 & 85 & $\mathbf{7 8}$ \\
\hline MK7 & $20 \times 5$ & 157 & 154 & $\mathbf{1 5 1}$ \\
\hline MK8 & $20 \times 10$ & $\mathbf{5 2 3}$ & $\mathbf{5 2 3}$ & $\mathbf{5 2 3}$ \\
\hline MK9 & $20 \times 10$ & 369 & 437 & $\mathbf{3 5 5}$ \\
\hline MK10 & $20 \times 15$ & 296 & 380 & $\mathbf{2 6 4}$ \\
\hline
\end{tabular}

\section{Conclusions}

In this paper, a modified bat algorithm name MBA is applied to solve the multi-objective FJSSP. In our proposed algorithm, the converting mechanisms are presented to make the continuous BA suitable for solving the discrete FJSSP. The initialization approaches integrating heuristic and random rules are introduced to initialize the population of the algorithm, which makes the MBA have good quality and diversity. To improve the searching ability, five neighborhood structures are designed, based on which a local search algorithm is hybridized with the BA.

According to the simulation results, MBA can acquire optimal or near-optimal solutions for the benchmark instances Some novel and effective neighborhood structures for the FJSSP should be developed for the local search. In addition, the application of BA to other kinds of combination optimization problems may also be a promising direction.

\section{Acknowledgements}

The authors would like to thank the editors and the anonymous reviewers for their helpful comments and suggestions, which have improved the presentation. This work was supported in part by the Science and Technology Plan Projects of Henan Province of China under grant No.152102210357 and No.152102210149, the Youth Backbone Teachers Funding Planning Project of Colleges and Universities in Henan Province of China under grant No.2014GGJS-084, the Key Science Research Project of Colleges and Universities in Henan Province of China under grant No.16A520030, the Youth Backbone Teachers Training Targets Funded Project of Zhengzhou University of Light Industry of Henan Province of China under grant No.XGGJS02, the Ph.D.Research Funded Project of Zhengzhou University of Light Industry of Henan Province of China under grant No.2010BSJJ038 and No.2014BSJJ080, and the National Science Foundation of China under grant No.81501548. 


\section{References}

1. A. Bagheri, M. Zandieh, I. Mahdavi, M. Yazdani, "An artificial immune algorithm for the flexible job-shop scheduling problem," Future Generation Computer Systems, vol. 26, no. 4, pp. 533-541, 2010.

2. P. Brandimarte, "Routing and scheduling in a flexible job shop by tabu search," Annals of Operations Research, vol. 41, no. 3, pp. 157-183, 1993.

3. K. Z. Gao, P. N. Suganthan, Q. K. Pan, "Pareto-based grouping discrete harmony search algorithm for multi-objective flexible job shop scheduling," Information Sciences, vol. 289, no. 7, pp. 76-90, 2014.

4. A. H. Gandomi, X. S. Yang, A. H. Alavi, S. Talatahari, "Bat algorithm for constrained optimization tasks," Neural Computing and Applications, vol. 22, no. 6, pp. 1239-1255, 2013.

5. A. Henchiri, M. Enngirou, "Particle swarm optimization combined with tabu search in a multi-agent model for flexible job shop problem,” Computer Science, vol. 7929, no. 1, pp. 385-394, 2013.

6. I. Kacem, S. Hammadi, P. Borne, "Approach by localization and multi-objective evolutionary optimization for flexible job-shop scheduling problems," IEEE Transactions on Systems, Man, and Cybernetics, Part C: Applications and Reviews, vol. 32, no. 1, pp. $1-13,2002$.

7. I. Kacem, S. Hammadi, P. Borne, "Pareto-optimality approach for flexible job-shop scheduling problems: hybridization of evolutionary algorithms and fuzzy logic," Mathematics and Computers in Simulation, vol. 60, no. 3, pp. 245-276, 2012.

8. V. Kaplanoğlu, "An object-oriented approach for multi-objective flexible job-shop scheduling problem," Expert Systems with Applications, vol. 45, no. 7, pp. 71-84, 2016.

9. J. Li, Q. Pan, Y. C. Liang, "An effective hybrid tabu search algorithm for multi-objective flexible job-shop scheduling problems," Computers \& Industrial Engineering, vol. 59, no. 4, pp. 647-662, 2010.

10. J. Q. Li, Q. K. Pan, M. F. Tasgetiren, "A discrete artificial bee colony algorithm for the multi-objective flexible job-shop scheduling problem with maintenance activities," Applied Mathematical Modelling, vol. 38, no. 3, pp. 1111-1132, 2014.

11. Q. Luo, Y. Zhou, J. Xie, et al, "Discrete bat Algorithm for optimal problem of permutation flow shop scheduling," The Scientific World Journal, vol. 25, no. 1, pp. 1-25, 2014.

12. M. K. Marichelvam, T. Prabaharam, "A bat algorithm for realistic hybrid flowshop scheduling problems to minimize makespan and mean flow time," ICTACT Journal on Soft Computing, vol. 3, no. 1, pp. 428-433, 2012.

13. M. K. Marichelvam, T. Prabaharan, X. S. Yang, et al, "Solving hybrid flow shop scheduling problems using bat algorithm," International Journal of Logistics Economics and Globalisation, vol. 5, no. 1, pp. 15-29, 2013.

14. G. Moslehi, M. Mahnam, "A Pareto approach to multi-objective flexible job-shop scheduling problem using particle swarm optimization and local search," International Journal of Production Economics, vol. 129, no. 1, pp. 14-22, 2011.

15. S. H. A. Rahmati, M. Zandieh, "A new biogeography-based optimization (BBO) algorithm for the flexible job shop scheduling problem," The International Journal of Advanced Manufacturing Technology, vol. 58, no. 12, pp. 1115-1129, 2012.

16. B. Ramesh, V. C. J. Mohan, V. C. V. Reddy, "Application of bat algorithm for combined economic load and emission dispatch," International Journal of Electricl Engineering and Telecommunications, vol. 2, no. 1, pp. 1-9, 2013.

17. M. Saidi-Mehrabad, P. Fattahi, "Flexible job shop scheduling with tabu search algorithms," The International Journal of Advanced Manufacturing Technology, vol. 32, no. 6, pp. 563-570, 2007.

18. N. Shivasankaran, P. S. Kumar, K. V. Raja, "Hybrid sorting immune simulated annealing algorithm for flexible Job shop scheduling," International Journal of Computational Intelligence Systems, vol. 8, no. 3, pp. 455-466, 2015.

19. ̈. Tosun, M. K. Marichelvam, "Hybrid bat algorithm for flow shop scheduling problems," International Journal of Mathematics in Operational Research, vol. 9, no. 1, pp. 125-138, 2016.

20. L. Wang, G. Zhou, Y. Xu, S. Wang, "An effective artificial bee colony algorithm for the flexible job-shop scheduling problem," The International Journal of Advanced Manufacturing Technology, vol. 60, no. 4, pp. 303-315, 2012.

21. S. Wang, C. Liu, D. Pei, J. Wang, "A novel hybrid election campaign optimisation algorithm for multi-objective flexible job-shop scheduling problem," International Journal of Materials and Structural Integrity, vol. 7, no. 1, pp. 160-170, 2013.

22. W. Xia, Z. Wu, "An effective hybrid optimization approach for multi-objective flexible job-shop scheduling problems," Computers \& Industrial Engineering, vol. 48, no. 2, pp. 409-425, 2005.

23. M. Yazdani, M. Amiri, M. Zandieh, "Flexible job-shop scheduling with parallel variable neighborhood search algorithm," Expert Systems with Applications, vol. 37, no. 1, pp. 678-687, 2010.

24. X. S. Yang, "A new metaheuristic bat-inspired algorithm," Springer Berlin Heidelberg, Germany,2010.

25. X. S. Yang, "Bat algorithm for multi-objective optimization," International Journal of Bio-Inspired Computation, vol. 3, no. 5, pp. 267-274, 2011.

26. Y. Yuan, H. Xu, J. Yang, “A hybrid harmony search algorithm for the flexible job shop scheduling problem,” Applied Soft Computing, vol. 13, no. 7, pp. 3259-3272, 2013.

27. G. Zhang, L. Gao, Y. Shi, "An effective genetic algorithm for the flexible job-shop scheduling problem," Expert Systems with Applications, vol. 38, no. 4, pp. 3563-3573, 2011. 